\title{
Estimation of traffic flow changes using networks in networks approaches
}

\author{
Jürgen Hackl ${ }^{*}$ (D) and Bryan T. Adey
}

\author{
*Correspondence: \\ hackl@ibi.baug.ethz.ch \\ Institute for Construction \\ Engineering and Management, ETH \\ Zurich, Stefano-Franscini-Platz 5, \\ 8093, Zurich, Switzerland
}

\begin{abstract}
Understanding traffic flow in urban areas has great importance and implications from an economic, social and environmental point of view. For this reason, numerous disciplines are working on this topic. Although complex network theory made their appearance in transportation research through empirical measures, the relationships between dynamic traffic patterns and the underlying transportation network structures have scarcely been investigated so far. In this work, a novel Networks in Networks (NiN) approach is presented to study changes in traffic flows, caused by topological changes in the transportation network. The NiN structure is a special type of multi-layer network in which vertices are networks themselves. This embedded network structure makes it possible to encode multiple pieces of information such as topology, paths, and origin-destination information, within one consistent graph structure. Since each vertex is an independent network in itself, it is possible to implement multiple diffusion processes with different physical meanings. In this way, it is possible to estimate how the travellers' paths will change and to determine the cascading effect in the network. Using the Sioux Falls benchmark network and a real-world road network in Switzerland, it is shown that NiN models capture both topological and spatial-temporal patterns in a simple representation, resulting in a better traffic flow approximation than single-layer network models.
\end{abstract}

Keywords: Networks in networks, Multi-layer networks, Network dynamics, Transportation, Infrastructure, Traffic flow, Diffusion, Simulation

\section{Introduction}

Mobility and accessibility are essential factors for lifestyle and prosperity. People travel to satisfy their needs, by carrying out certain activities at specific places such as work, leisure and learning. The spatial distribution of these activities often leads to a coordination problem, which can significantly affect the equilibrium between the demand for, and the supply of transportation. To determine network flow, costs and other aspects of interest, the satisfaction of a given demand for movements of persons and goods with different trip purposes, at different times, using various modes of transport, must be ensured in a transport system with a given operational capacity (de Dios Ortuozar and Willumsen 2011). As a result of the dynamic nature between mobility demand and supply, in combination with the topology and capacity limitation of the underlying network, transportation networks exhibit atypical dynamic behaviour. Unlike many other networks, network performance

(c) The Author(s). 2019 Open Access This article is distributed under the terms of the Creative Commons Attribution 4.0 International License (http://creativecommons.org/licenses/by/4.0/), which permits unrestricted use, distribution, and reproduction in any medium, provided you give appropriate credit to the original author(s) and the source, provide a link to the Creative Commons license, and indicate if changes were made. 
deteriorates as soon as the number of vehicles in the network exceeds a critical accumulation (Daganzo and Geroliminis 2008; Hoogendoorn and Knoop 2012), i.e. vehicles block each other and the flow decreases, leading to spillbacks and gridlock effects. This phenomenon is amplified by the fact that even small (unexpected) failures or damage to the infrastructure (i.e. changes in topology) can lead to significant disruptions that are disproportionate to the actual physical damage itself (Vespignani 2010). To prevent such situations, scientists and engineers are working on the implementation of resilient systems capable of withstanding failures, natural hazards and human-made disruptions. Part of the research deals with the quantification of network-related risks, including the modelling of traffic flows after multiple link failures (Erath 2011; Hackl et al. 2018a; Hackl et al. 2018b).

Traffic models are needed to simulate the current and predict future traffic flows. An essential component of such models is the so-called traffic assignment process, which aims to reproduce the pattern of vehicular movements based on certain behavioural rules (Wang et al. 2018). For example, a common behavioural rule is that travellers choose paths with minimum travel time (Wardrop 1952) or maximise their utilities (Charypar and Nagel 2005). In order to satisfy the needs of all travellers, an equilibrium between demand and supply has to be found, i.e. no traveller wants to change his path. This complex and computationally intensive mathematical problem is still being actively researched. To make matters worse, in order to quantify network-related risks, resilience, or optimal intervention strategies, the traffic assignment problem must not only be solved once but many times with different network topologies (e.g. see (Erath 2011; Vugrin et al. 2014; Hackl et al. 2018a; Hackl et al. 2018b; Schlögl et al. 2019)).

While addressing such problems have led to a substantial body of work in areas such as geography, economics, and transportation research, complex network theory still plays a minor role. Although complex networks made their appearance in transportation research through empirical measures, little research has so far been done to investigate the relationship between dynamic traffic patterns and the underlying structures of the transportation networks (Barrat et al. 2008).

In this work, the application of a novel Networks in Networks (NiN) approach is presented. This approach is used to study traffic flow changes caused by topological changes in the transportation network (e.g. due to multiple link failures) from a complex network perspective. NiNs are based on a multi-layer approach where each vertex itself represents a network. This embedded network structure allows encoding multiple pieces of information such as the topology, paths used and origin-destination information, within one consistent graph structure (i.e. using only vertices and edges). In combination with a multi-layered diffusion process, an approximation to changes in traffic flow due to topology changes can be made. Specifically, this work advances the state-of-the-art in the field of complex network science in transportation research as follows.

- Using a modified multi-layer hypergraph it is formally feasible to describe vertices that are networks themselves. Thereby the relationships in the incidence graph represent the edges connecting different layers. The edges within the different layers are given by a connection model, which allows different topologies in the different layers.

- Because each vertex is an independent network in itself, it is possible to implement multiple diffusion processes. Therefore, it is possible to assign different physical 
meanings to the processes. For example, one process can describe how individual travellers switch between different paths, while another process describes the propagation of disturbances through the network.

- The proposed approach allows approximating traffic flow changes due to multiple edge failures. Using the Sioux Falls benchmark network and a real-world road network in Switzerland, it is shown that NiN models capture both topological and spatial-temporal patterns in a simple representation, resulting in a better traffic flow approximation than single-layer network models.

This work is organised as follows. A brief overview of the modelling, functionality and complexity of transport systems is given in the following section. In addition, advances in complex network theory regarding transport systems and dynamic processes are discussed. A general formulation for the NiN representation is presented in the "Methodology" section. In addition, the application to transportation networks and the modelling of traffic flow changes are discussed on a general level. Two applications are presented, the modelling of a small benchmark network and the modelling of a real network located in Switzerland. In particular, this section is divided into an overview of the data used, the assumptions made and the implementation of the methodology. Subsequently, the results and a critical discussion about the results, advantages and disadvantages of the method are given. Finally, concluding remarks and suggestions for further work in this area are presented. The notation used in this work is listed in the appendix.

\section{Background}

\section{Transportation networks (preliminaries)}

The purpose of transport systems is to balance supply and demand for mobility. The demand for transport is derived from people trying to satisfy their needs (work, leisure, health, education) through activities in specific places. Transport supply is the service provided at a certain point in time. This includes the infrastructure (e.g. road network) and a set of mobile units (e.g. persons, vehicles, goods). In combination with a set of rules for operation, the movements of persons and goods can be ensured (de Dios Ortuozar and Willumsen 2011). In order to predict how the need for mobility will manifest itself in space and time, a formal representation of the transport system is required. In a mathematical sense such systems are often represented as graphics or networks, which are denoted by $G=(\mathcal{V}, \mathcal{E})$ and consist of a set of edges $\mathcal{E}$ and a set of vertices $\mathcal{V}$. In this work, the term infrastructure network is used to refer to networks where only topology and connectivity are considered (Rodrigue et al. 2009), i.e. the network comprises vertices and edges that form a connected component. If, in addition to topology, flow characteristics, such as origin-destination demands, capacity constraints, path choice and travel costs, are taken into account to represent the movement of people, vehicles or goods, the network is referred to as transportation network.

In a transportation network, the edges represent the movement between vertices, which in turn represent points in space. An edge $e \in \mathcal{E}$ connects two vertices $v_{i}, v_{j} \in \mathcal{V}$ and a vertex connects two or more edges. Edges can be either directed $e=\left(v_{i}, v_{j}\right) \in \mathcal{E}$, indicating that $v_{i}$ and $v_{j}$ are directly connected and movement is only possible from $v_{i}$ to $v_{j}$, or undirected $\left(e=\left\{v_{i}, v_{j}\right\} \in \mathcal{E}\right)$. Important properties of transportation network edges include edge length, edge cost and edge capacity. The edge length corresponds to the length of the 
road section connecting two vertices. The term edge cost is used to describe the disutility perceived by the network user for travelling on this edge. It is a composite measure of all factors known to be important for decision-making. Travel time and direct costs such as fuel consumption, parking fees and tolls are often taken into account for this purpose. Since transportation networks are physically constrained, it is assumed that each edge has a maximum capacity, i.e. a maximum rate at which people, vehicles or goods can travel on an edge during a given period under prevailing roadway, traffic and operation conditions (Hoogendoorn and Knoop 2012). The movements in a transportation network correspond to flows with a distinct origin and destination. Origins and destinations can represent particular locations such as residential buildings, offices, shopping centres, or specific zones. In the context of transportation networks, origins and destinations are represented as vertices $o, d \in \mathcal{V}$. It should be noted that not all vertices in the network need to be an origin or a destination. Vehicle movements from origin $o$ to destination $d$ vertices occurring along edges are represented as paths. A path $p \in \mathcal{P}$ is considered as a sequence of edges that ordered so that two vertices are adjacent if and only if they are consecutive. $\mathcal{P}$ therefore denotes the set of all simple non-empty paths in $G=(\mathcal{V}, \mathcal{E})$. The set of $o d$-paths is denoted by $\mathcal{P}_{o d} \subseteq \mathcal{P}$.

To estimate the movements in a transportation network, it is necessary to find an equilibrium between demand and supply, i.e. in the equilibrium situation, the user chooses the path that he perceives to be the least costly at the time. Economic theory admits that this equilibrium may never really actually occur in practice, as the system of demand and supply levels is constantly adapting to cope with internal and external changes. However, the concept of equilibrium is still valuable to understand movement in transportation networks, assuming that the system is at least near an equilibrium situation. In order to find this equilibrium, various traffic flow models have been developed in recent decades. The most common classification in current traffic flow research is the distinction between macroscopic and microscopic traffic flow modelling approaches (Hoogendoorn and Knoop 2012). The macroscopic perspective considers the overall or average state of traffic, while the microscopic perspective considers the behaviour of individuals interacting with surrounding vehicles. Macroscopic models were the first to be derived by scientists (Wardrop 1952; Lighthill and Whitham 1955) who studied vehicle flow as an analogy to the flow of continuous media such as fluids or gases. These models are based on a limited number of partial differential equations, which reduces the computational complexity. The disadvantage, however, is that dynamic features cannot be modelled as accurate as with microscopic models. Microscopic models have been developed to try to emulate human behaviour in traffic situations. To accomplish this, the models contain different driving conditions to describe typical driving reactions. As each vehicle is an autonomous entity, microscopic models become very computationally expensive with increasing system size.

In order to reduce the computational time for both modelling approaches, scholars have developed various techniques. This includes among others, the improvement of the optimisation algorithms to find an equilibrium solution (Charypar and Nagel 2005; Mitradjieva and Lindberg 2013; Gentile 2014); the development of speed-up techniques for sub-problems of the traffic assignment (e.g. finding the shortest paths) (Geisberger et al. 2008; Delling et al. 2009; Buchhold et al. 2018) or the utilisation of GPU cards to parallelise (agent-based microscopic) traffic models (Song et al. 2017; Heywood et al. 2018). 
Another way to address these problems could be through the use of complex network approaches.

\section{Traffic on complex networks}

Complex networks are based on the ideas of mathematical graph theory in order to gain insights into the behaviour of complex systems by abstracting information into ordinary graphs (networks). In these representations, the network comprises vertices connected by edges, with vertices representing individual elements and edges indicating interactions or relationships between them. Although this approach is simple in many ways, it allows the characterisation of the complex system so that traditional graphic-theoretical metrics can be used and analyses performed. For example, such abstractions have been used to study growth mechanisms (Barabási and Albert 1999; Clauset et al. 2009), processes of collective dynamics (Watts and Strogatz 1998), and to illustrate that certain vertices play a central role in the complex system (Freeman 1977; Wasserman and Faust 1994).

The strength of the complex network paradigm lies in its ability to capture some of the essential structural features of interacting systems while reducing the details of both the elements and their interactions. Consequently, the early complex network literature focused almost exclusively on the structural properties of networks Smith et al. (2011). This topology-driven analysis can reveal relevant properties of the structure of a complex system (Albert et al. 1999; Watts and Strogatz 1998) by highlighting the role of vertices and edges (Bavelas 1950; Freeman 1977) or global network properties (Taaffe et al. 1973; Cliff et al. 1979). The robust mathematical framework allows the derivation of analytical solutions even for large complex systems. For example, hierarchical network representations are used to study large complex transportation systems (Gómez et al. 2013; Lim et al. 2015). Thereby, hierarchical models are obtained by successive clustering of networks, i.e. decomposition of the system into different levels of details (Ferrario et al. 2016).

While structural properties are still important in constraining the behaviour of a system (Marr and Hütt 2005), the focus has expanded to an understanding of the relationship between structure and dynamics that takes place in networks and the impact of this relationship on network design (Toroczkai 2005). Most technological, biological, economic, social or infrastructural networks support a number of dynamic (transport) processes, such as the movement of information packages (Wang et al. 2006), finance and wealth (Coelho et al. 2005), rumours (Moreno et al. 2004), diseases (Newman 2002), people or goods. Gradually, these theories have been introduced to the field of transportation. More and more scholars have conducted research on the characteristics of various transportation networks, among others those of (urban) road networks (De Montis et al. 2007; Erath et al. 2009; Barthélemy 2011; Lin and Ban 2013), railway networks (Latora and Marchiori 2002; Sen et al. 2003), and transit networks (Guo Xl and Lu 2016; Solé-Ribalta et al. 2016). In addition, current studies use complex networks to analyse traffic time series (Tang et al. 2013; Yan et al. 2017; Bao et al. 2017).

In the field of complex network sciences, a widely used approach to study the relationship between dynamic processes and the underlying network structures is through the use of random walks. In such a model, random walkers move in the network and visit various edges and vertices over time. An extensive overview of the use of random walks and diffusion on complex networks is given by Masuda et al. (2017). Researchers, using 
this technique, have been able to gain insights into topological features such as vertex centralities (Brin and Page 1998) or community structures (Newman 2006; Jeub et al. 2015).

In many real transportation systems, however, the assumption of such simple random walker models may not always be justified, since it is assumed that the walker moves randomly in the network without considering its origin or destination. For example, in a diffusion process on a road network, the next position of a vehicle depends only on its current position (occupied vertex) and the outgoing roads (edges), but not on one of the previously visited locations. In reality, however, travel in a network has a specific purpose: a person starts at home and navigates through the network to reach a particular destination (e.g. work), and then returns home with a high probability (Salnikov et al. 2016). Consequently, the naive application of (static) network paradigm in modelling dynamic complex systems might lead to wrong conclusions (Rosvall et al. 2014; Scholtes et al. 2014; Scholtes 2017). One way to address this issue is through the extension to a multi-layer networks representation.

Multi-layer networks represent complex systems that are formed from several networks (layers), each of which represents interactions of different nature and connections. Due to the distinction between the different types of edges and vertices, multi-layer networks encode significantly more information than conventional single-layer networks (Iacovacci and Bianconi 2016). In network science, the two most prominent classes of multi-layer networks are multiplex networks and networks of networks. Networks of networks are formed by layers composed of different vertices. Edges connecting different networks do not necessarily indicate dependency relationships. Examples can be found in complex infrastructure networks such as road networks, railway networks and flight networks, where each layer represents its own infrastructure. Multiplex networks, on the other hand, are formed by the same set of vertices connected by edges indicating different types of interactions. In the context of transport systems, this approach has been used, for example, for the analysis of flight networks (Cardillo et al. 2013). An overview of other types of multi-layer networks is given by Boccaletti et al. (2014); Kivelä M et al. (2014); Bianconi (2018). In all these definitions, it is assumed that a multi-layer network can be represented as a graph $G_{M}$, which is an ordered tuple $G_{M}=\left(\mathcal{V}_{M}, \mathcal{E}_{M}\right)$ considering a nonempty labelled vertex set $\mathcal{V}_{M}$ and a multiset $\mathcal{E}_{M} \subseteq \mathcal{V}_{M} \times \mathcal{V}_{M}$ of edges. A vertex $v^{\alpha} \in \mathcal{V}_{M}$ is a tuple representing vertex $v$ on the layer $\alpha \in \mathcal{L}$, where $\mathcal{L}$ is the set of layers in the network.

\section{Methodology}

\section{Networks in networks}

A Networks in Networks (NiN) structure is a special type of multi-layer network in which vertices themselves are networks, i.e. $G_{\mathrm{NiN}}=\left(\mathcal{G}_{\mathrm{NiN}}, \mathcal{E}_{\mathrm{NiN}}\right)$ is a network with a set of graphs $\mathcal{G}_{\mathrm{NiN}}$ acting as vertices and a multiset $\mathcal{E}_{\mathrm{NiN}} \subseteq \mathcal{G}_{\mathrm{NiN}} \times \mathcal{G}_{\mathrm{NiN}}$ of edges. A vertex $v_{i}^{\beta} \in \mathcal{G}_{\beta}$ is a tuple representing a graph $v_{i}^{\beta}:=\left(\mathcal{G}_{\alpha}, \mathcal{E}_{\alpha}\right)$, where $\alpha<\beta ; \alpha, \beta \in \mathcal{L}$ are the layers describing the order of hierarchy in the network. It should be noted that the vertices for each layer are defined recursively since they are constructed from a previous layer.

The set of vertices $\mathcal{G}_{\mathrm{NiN}}$ can be interpreted as a modified multi-layer hypergraph $H_{M}=$ $\left(\mathcal{X}_{M}, \mathcal{Y}_{M}\right)$, where $\mathcal{X}_{M}$ is a set of basic elements and $\mathcal{Y}_{M}$ is a set of non-empty subsets of $\mathcal{X}_{M}$, here denoted as hypervertices. ${ }^{1}$ Hypervertices are arbitrary sets of vertices and can contain therefore any number of vertices. In a multi-layer structure, higher-order 
hypervertices are arbitrary sets of lower-order hypervertices, whereby the order indicates the position in a hierarchical layered structure.

$$
\bigcup_{j=1}^{n^{\beta}} y_{j}^{\beta}=\bigcup_{i=1}^{n^{\alpha}} y_{i}^{\alpha} \quad \text { with } \quad \alpha<\beta ; \alpha, \beta \in \mathcal{L}
$$

where $\mathcal{Y}_{\alpha}:=\left(y_{i}^{\alpha}\right)_{i \in \mathcal{I}^{\alpha}}$ with index set $\mathcal{I}^{\alpha}$ and $n^{\alpha}$ is the number of hypervertices in layer $\alpha$. The same is true for layer $\beta$. In the layer with the lowest order, the hypervertices are sets of the basic elements:

$$
\bigcup_{i=1}^{n^{\alpha}} y_{i}^{\alpha}=\mathcal{X} \quad \text { with } \quad \alpha=\min \{\alpha, \beta, \ldots\} \in \mathcal{L}
$$

The relationships between the different types of hypervertices are described by their incidence structure. Unlike the classical incidence graph associated with the hypergraph, the incidence graph for a NiN consists of inter-layer edges, which define the relationship between hypervertices of different orders. The intra-layer edges, which describe edges within a layer, are defined by adjacency matrices for each layer. The incidence matrix between two consecutive layers $\alpha<\beta, \alpha, \beta \in \mathcal{L}$ is given by $\vec{I}^{\alpha \beta}=i_{i j}^{\alpha \beta}$ where:

$$
i_{i j}^{\alpha \beta}=\left\{\begin{array}{l}
1 \text { if } y_{i}^{\alpha} \in y_{j}^{\beta} \\
0 \text { otherwise }
\end{array} \quad \text { with } \quad i=1, \ldots, n^{\alpha} ; \quad j=1, \ldots, n^{\beta} .\right.
$$

The adjacency matrix of each layer $\alpha \in \mathcal{L}$ is given by $\vec{A}^{\alpha}=a_{i j}^{\alpha}$ where:

$$
a_{i j}^{\alpha}=\left\{\begin{array}{l}
1 \text { if }\left(v_{i}^{\alpha}, v_{j}^{\alpha}\right) \in \mathcal{E}_{\alpha} \quad \text { with } \quad v_{i}^{\alpha}, v_{j}^{\alpha} \in \mathcal{G}_{\alpha} . \\
0 \text { otherwise }
\end{array}\right.
$$

Considering that hypervertices itself are networks, a transformation into a classical supra-adjacency matrix (Boccaletti et al. 2014; Kivelä M et al. 2014; Cozzo et al. 2016), (i.e. the representation of a multi-layer network as a single-layer network), may not always be possible or intended because information is lost in the transformation process (Kivelä $M$ et al. 2014).

\section{Connection models}

In order to consider the influence of the different lower-order hypervertices on the connection between two hypervertices, connection models are used for the intra-layer edge assignment (Meester and Roy 1996; Hackl and Adey 2017; 2018). A connection model $\mathcal{M}\left(\mathcal{G}_{\beta}, g_{\beta}\right)$ has two characteristics. First, the set of vertices $\mathcal{G}_{\beta}$ which should be connected and second the so-called connection function $g_{\beta}$, which gives the probability of a direct link between two vertices $v_{i}^{\beta}$, and $v_{j}^{\beta}$ on layer $\beta$ (Meester and Roy 1996). Since the connection function can be any arbitrary functional relationship between two vertices, it allows to define the entries of the adjacency matrices for each layer depending on the network properties of the embedded vertices.

Formally, the multiset $\mathcal{E}_{\beta}$ can be defined as a connection model $\mathcal{E}_{\beta}:=\mathcal{M}\left(\mathcal{G}_{\beta}, g_{\beta}\right)=$ $\left\{\left(v_{i}^{\beta}, v_{j}^{\beta}\right) \mid g\left(v_{i}^{\beta}, v_{j}^{\beta}\right) ; v_{i}^{\beta}, v_{j}^{\beta} \in \mathcal{G}_{\beta}\right\}$, with the connection function $g: v_{i}^{\beta} \times v_{j}^{\beta} \rightarrow\{0,1\}$, which is a mapping from the underlying graphs $v_{i}^{\beta}=\mathcal{G}_{\alpha_{i}}$ and $v_{j}^{\beta}=\mathcal{G}_{\alpha_{j}}$ to 0 (not connected) or 1 (connected) with $\alpha<\beta ; \alpha, \beta \in \mathcal{L}$. In addition, $g$ can be a mapping to $\mathbb{R}^{+}$ 
that indicates the weight of the edge $\left(v_{i}^{\alpha}, v_{j}^{\alpha}\right)$. An overview of several connection functions is given by Dettmann and Georgiou (2016); Parsonage and Roughan (2017); Hackl and Adey (2018).

\section{NiN structure for transportation networks}

The following section presents considerations on how a NiN model can be used for transportation networks. An important point to consider is the concept of origin and destination. Therefore, the network representation should contain information of an origin-destination matrix as well as the path statistics, i.e. how many trips from $v_{i}$ to $v_{j}$ are taken and which paths are used therefore? To achieve this, a NiN network with four layers is presented. The first layer $(\alpha)$ consists of the vertices of the infrastructure network, where the basic elements correspond to the intersections of the transport network. In the second layer $(\beta)$ possible paths of the network are mapped. These are grouped in the third layer $(\gamma)$ according to their origins and destinations. In the last layer $(\delta)$ these origin-destination paths are combined to describe all trips in the network. A conceptual illustration of a simple transportation network is given in Fig. 1. The individual layers are described in more detail below, with references to Fig. 1.

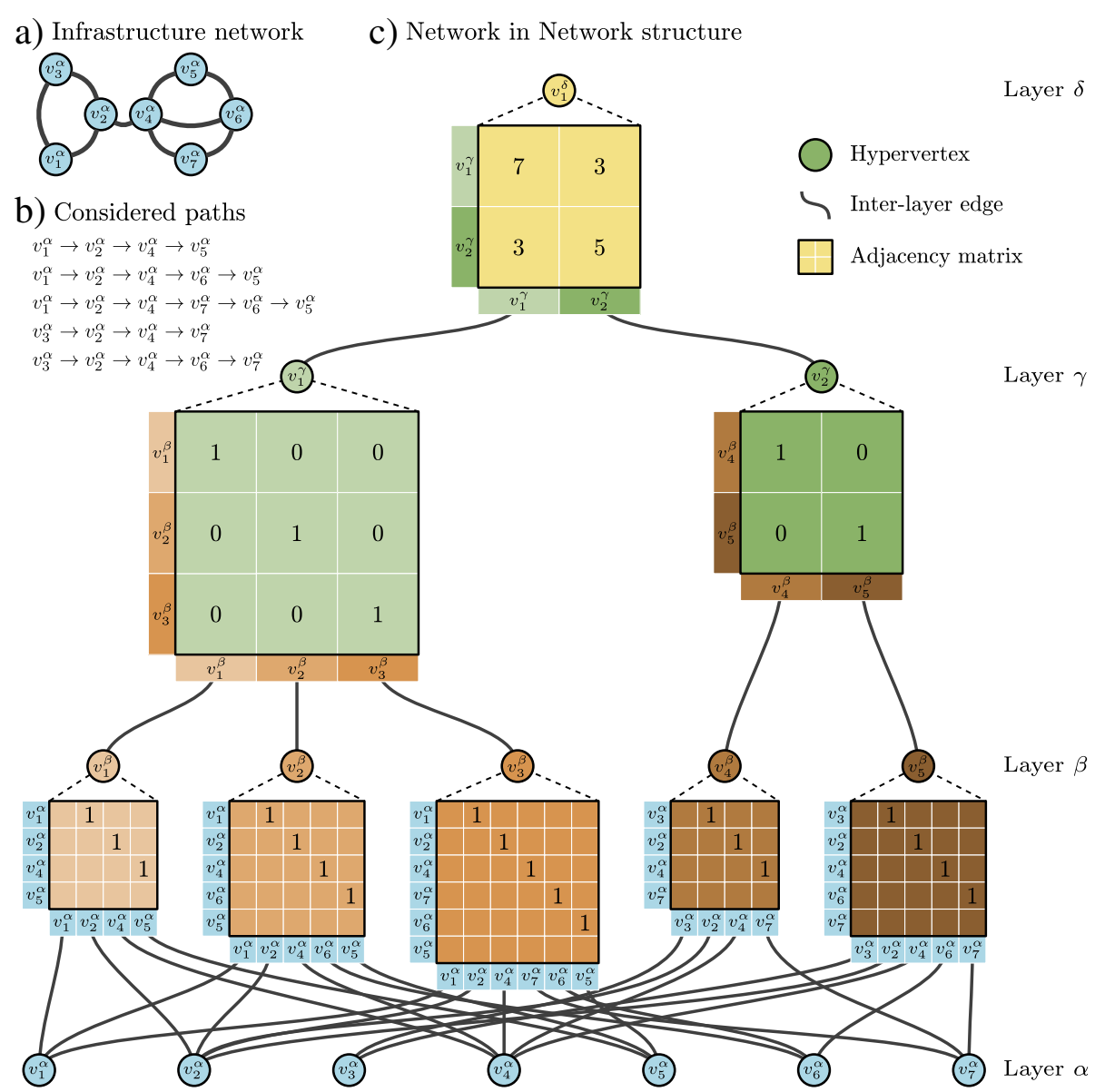

Fig. 1 NiN structure for transportation network example. a Visualisation of the underlying infrastructure network. $\mathbf{b}$ Considered paths in the network. $\mathbf{c}$ Incidence graph of the NiN model with the associated adjacency matrices of the hypervertices 
Layer $\alpha$. The underlying infrastructure network comprises vertices and edges. Vertices correspond to objects or locations in the physical world such as road junctions or facilities. Edges connecting these vertices represent road sections. The basic elements of the NiN model are the vertices of the underlying infrastructure network. Based on these vertices, all other layers of the NiN are constructed. For example, the set of basic elements for the infrastructure network in Fig. 1 is given by $\mathcal{X}=\left\{v_{1}^{\alpha}, v_{2}^{\alpha}, v_{3}^{\alpha}, v_{4}^{\alpha}, v_{5}^{\alpha}, v_{6}^{\alpha}, v_{7}^{\alpha}\right\}$ [Eq. 2].

Layer $\beta$. Considered paths $\mathcal{P}$ in the network are described in the second layer. Each path is represented as a hypervertex $v_{i}^{\beta} \in \mathcal{G}_{\beta}$, composed of a set of connected basic elements. The hypervertex $v_{i}^{\beta}$ is a graph that describes a sequence of vertices connected by edges in one direction, so that movement from the first vertex to the last vertex is possible. Obviously, edges can only be assigned if they are also present in the underlying infrastructure network. In Fig. 1 five paths are considered. Path $v_{1}^{\beta}$, for example, connects vertex $v_{1}^{\alpha}$ with vertex $v_{5}^{\alpha}$ via $v_{1}^{\alpha} \rightarrow v_{2}^{\alpha} \rightarrow v_{4}^{\alpha} \rightarrow v_{5}^{\alpha}$ and is defined as $v_{1}^{\beta}:=\left(\left\{v_{1}^{\alpha}, v_{2}^{\alpha}, v_{4}^{\alpha}, v_{5}^{\alpha}\right\},\left\{\left(v_{1}^{\alpha}, v_{2}^{\alpha}\right),\left(v_{2}^{\alpha}, v_{4}^{\alpha}\right),\left(v_{4}^{\alpha}, v_{5}^{\alpha}\right)\right\}\right)$. The first set represents a subset of the lower-order hypervertices [Eq. 1]. The relationship between different orders of hypervertices (inter-layer edges) is described by an incidence matrix [Eq. 3] or an incidence graph, as depicted in Fig. 1. The second set represents the connections among the lowerorder hypervertices (intra-layer edges), represented in Fig. 1 as adjacency matrices [Eq. 4]. Indices of the adjacency matrices are the lower-order hypervertices.

Layer $\gamma$. Paths with the same origin and destination are grouped in layer $\gamma$. In other words, each hypervertex $v_{i}^{\gamma} \in \mathcal{G}_{\gamma}$ comprises a set of paths $v_{j}^{\beta} \in \mathcal{G}_{\beta}$ with the same orign destination vertices $v_{o}^{\alpha} \rightarrow \cdots \rightarrow v_{d}^{\alpha}$, where $v_{o}^{\alpha}$, $v_{d}^{\alpha} \in \mathcal{G}_{\alpha}$. The connection between the vertices can be interpreted as the choice a traveller has, in order to switch from the initially assigned path $v_{i}^{\beta}$ to any other path available between the origin and the destination, including self-loops where he remains on his initial path. The edge weighting corresponds to the probability of changing the path, i.e. the adjacency matrix is a direct representation of the transition matrix. For example, in the situation of an equilibrium between supply and demand, no one will alter their path choice and therefore only self-loops with weight 1 are observed, as illustrated in Fig. 1. In order to consider the influence of lower-order hypervertices and their properties (e.g. interrupted or congested paths), connection models can be used for the intra-layer edge and weight assigment. Thus hypervertex $v_{1}^{\gamma}$ can be expressed as $v_{1}^{\gamma}=\left(\left\{v_{1}^{\beta}, v_{2}^{\beta}, v_{3}^{\beta}\right\},\left\{\left(v_{i}^{\beta}, v_{j}^{\beta} \mid g\left(v_{i}^{\beta}, v_{j}^{\beta}\right) ; v_{i}^{\beta}, v_{j}^{\beta} \in \mathcal{G}_{\beta}\right)\right\}\right)$, where an edge is assigned if the criteria of the connection function $g$ are fulfilled (e.g. assign an edge with weight 1 if $v_{i}^{\beta}=v_{j}^{\beta}$, otherwise not).

Layer $\delta$. The top layer combines all origin-destination paths, similar to an origindestination matrix. In contrast to the classical matrix representation, where each cell represents the number of trips from the origin (row) to the destination (column), the hypervertex $v_{i}^{\delta} \in \mathcal{G}_{\delta}$ comprises all paths $v_{j}^{\gamma} \in \mathcal{G}_{\gamma}$, grouped by their origin-destination vertices. Furthermore, this lower-order hypervertices contain detailed path information $v_{k}^{\beta} \in \mathcal{G}_{\beta}$ derived from the underlying basic elements $v_{l}^{\alpha} \in \mathcal{G}_{\alpha}$. Edges in this layer can have different meanings. In this work, it is assumed that the edges represent the common road sections between different origin-destination paths. For example in Figure 1, travellers from $v_{1}^{\alpha}$ to $v_{5}^{\alpha}$ share three common road segments with the travellers from $v_{3}^{\alpha}$ to $v_{7}^{\alpha}$. The edges and weights can also be assigned using a connection model (e.g. assign an edge with weight $n\left(E_{\beta \mid v_{i}^{\gamma}} \wedge E_{\beta \mid v_{j}^{\gamma}}\right)$ if paths in $v_{i}^{\gamma}$ and $v_{j}^{\gamma}$ 
share common edges, where $E_{\beta \mid v_{i}^{\gamma}}$ is the set of edges in layer $\beta$ associated with hypervertex $v_{i}^{\gamma}$ and $n(\cdot)$ is a cardinality operator. In Figure 1, for example, $W\left(v_{1}^{\gamma}, v_{1}^{\gamma}\right)=$ $\left.n\left(\left\{\left(v_{1}^{\alpha}, v_{2}^{\alpha}\right),\left(v_{2}^{\alpha}, v_{4}^{\alpha}\right),\left(v_{4}^{\alpha}, v_{5}^{\alpha}\right),\left(v_{5}^{\alpha}, v_{6}^{\alpha}\right),\left(v_{6}^{\alpha}, v_{5}^{\alpha}\right),\left(v_{4}^{\alpha}, v_{7}^{\alpha}\right),\left(v_{7}^{\alpha}, v_{6}^{\alpha}\right)\right\}\right)=7.\right)$

\section{NiN dynamics for traffic flow changes}

Once the NiN structure has been created, dynamic processes on the network can be studied. Among other things, it is important to understand how the system behaves when several road sections fail, i.e. how do the affected trips change and does they have an influence on a global level? To study such behaviour, diffusion processes on two layers of the $\mathrm{NiN}$ model are applied. On layer $\gamma$ this is used to estimate how the path choices of the travellers are changing, while on layer $\delta$ the strength of the cascading effect is determined. This process is schematically illustrated in Fig. 2 and described in more detail below.

In the first phase, a set of interrupted edges $\overline{\mathcal{E}}_{\alpha} \subseteq \mathcal{E}_{\alpha}$ is removed from the considered paths $v_{i}^{\beta} \in \mathcal{G}_{\beta}$. This interrupts the sequence of vertices connected by edges, so that no

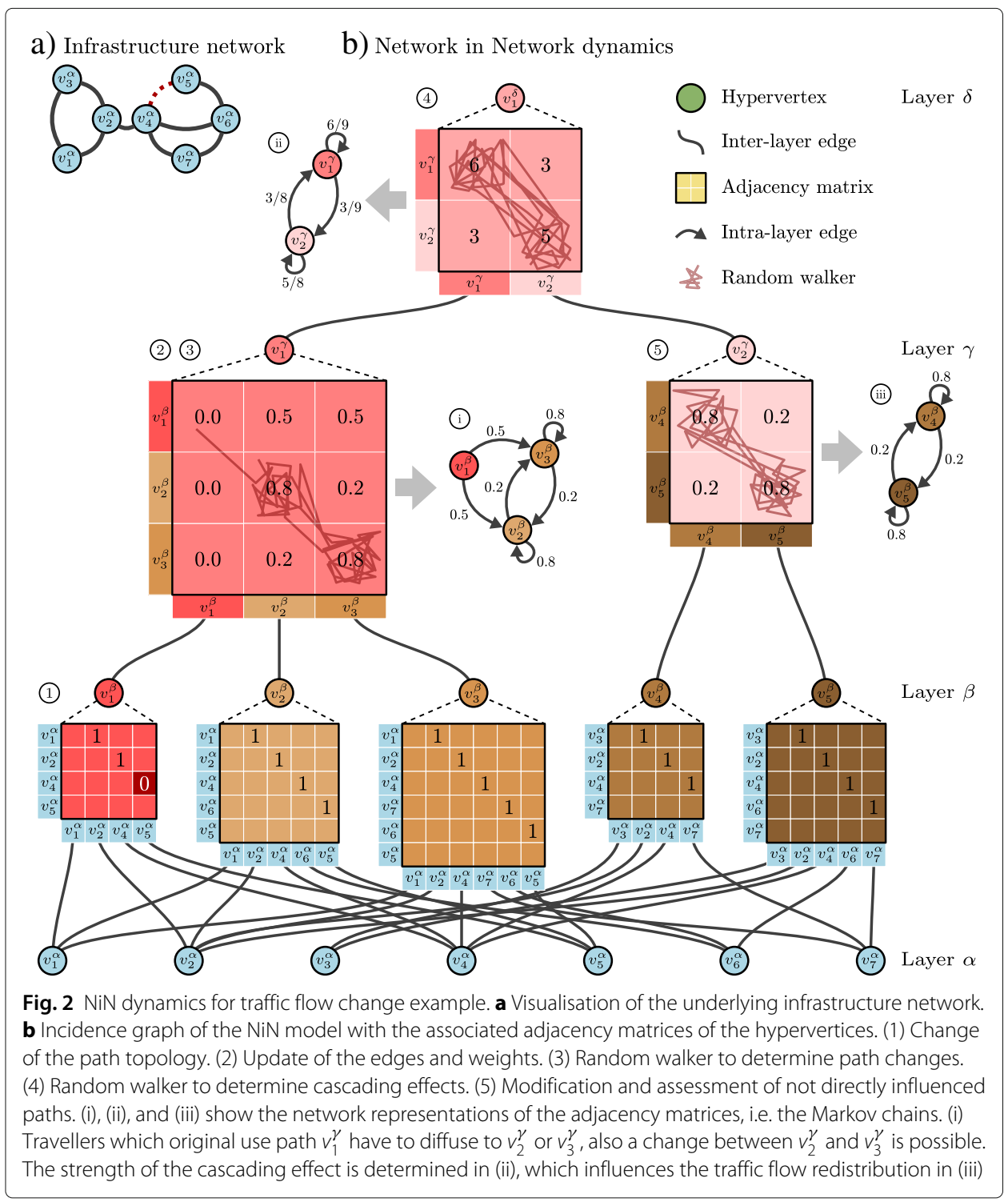


movement from the first to the last vertex is possible, i.e. with the number of edges available, the path cannot be completed $\left(n\left(\mathcal{E}_{\alpha}\right) \leq n\left(\mathcal{G}_{\alpha}\right)-2\right)$. In Fig. 2, for example, it is assumed that the edge $\left(v_{4}^{\alpha}, v_{5}^{\alpha}\right)$ is interrupted. Hence, path $v_{1}^{\beta}$, containing this edge, can no longer be used to reach the desired destination $v_{5}^{\alpha}$.

As a first consequence, people, vehicles or goods that initially used these paths must be redistributed to other possible paths. The likelihood of changing to another path is determined by the weights of the adjacency matrix of $v_{i}^{\gamma} \in \mathcal{G}_{\gamma}$. Using an extension of the previously introduced connection model, the edge and weight assignment can be done based on the properties of the lower-order hypervertices (e.g. if the underlying path is interrupted, assign edges to other paths with equal weights). Since in Fig. 2 path $v_{1}^{\beta}$ is interrupted, travellers have either to use path $v_{2}^{\beta}$ or $v_{3}^{\beta}$. Here, it is assumed that these travellers distribute equally on the remaining paths. Furthermore, travellers from neighbouring paths might also reconsider their path choices resulting in $20 \%$ additional path changes.

The behaviour of changing paths based on an underlying transition matrix can be described by a diffusion process and modelled with the help of random walkers. In this context, a random walker represents a traveller who randomly decides which path to take, given some preferences (e.g. edge weights). In the case of an interrupted path, the random walker remains with zero probability and visits instead another path. This stochastic process can be described as a sequence of random variables $X_{0}, X_{1}, \ldots, X_{m}$, where $X_{m}$ denotes the position of the random walker in the network at step $m$. Assuming that each movement simulated in the network is only dependent on the current position, the problem is reduced to a first-order Markov model:

$$
\mathbb{P}\left(X_{m} \mid X_{m-1}, X_{m-2}, \ldots, X_{0}\right)=\mathbb{P}\left(X_{m} \mid X_{m-1}\right)
$$

Thus, all this information can be captured by the transition matrix $\vec{T}$ :

$$
\mathbb{P}\left(X_{m}=v_{j} \mid X_{m-1}=v_{i}\right)=\vec{T}_{i j}=t_{i j}=\frac{W\left(v_{i}, v_{j}\right)}{\sum_{k} W\left(v_{i}, v_{k}\right)}
$$

measuring the probability that a random walker at vertex $v_{i}$ will go to vertex $v_{j}$ considering the connection strengths ${ }^{2}$. These connection strengths indicate the preferences of the traveller to choose one path over another. If $m \rightarrow \infty$ and the Markov chain is ergodic, a unique stationary distribution $\pi^{\beta}$ can be observed, i.e. $\pi^{\beta} \vec{T}=\pi^{\beta}$. This vector can be interpreted as a new equilibrium distribution of the selected paths given a set of interrupted paths. Multiplied with the initial observed traffic flows $f_{i}^{\beta}$ on paths $v_{i}^{\beta}$, the traffic flows $\hat{f}_{i}^{\beta}$ in the interrupted network can be estimated. A simple trajectory of a random walker on $v_{1}^{\gamma}$ is illustrated in Fig. 2. The walker starts on the interrupted path $v_{1}^{\beta}$ and moves to the other paths, where he stays with probability of $80 \%$.

This redistribution of path flows can cause cascading effects since the road sections of the new paths are also used by others. In order to consider these interdependencies, a second diffusion process is modelled in the uppermost layer $\delta$. In this layer, edges represent the connections between different origin-destination paths, weighted with the number of common road sections. In this work, it is assumed that this weight indicates how strongly different origin-destination paths influence each other, i.e. paths which share no common road sections will not (directly) influence each other, while paths which share almost all their road sections will have a strong influence on each other. Similar to the previous layer, 
a diffusion process is used to determine the propagation of the cascading effect. The stationary distribution $\pi^{\gamma}$ indicates how strongly other vertices are affected, even if they do not contain paths with interrupted edges.

In such a case, the diffusion process on layer $\beta$ is repeated, however, the new distribution of path flows is a combination of initial distribution and the redistribution, weighted by the mapped strength of the cascading effects $h\left(\pi^{\gamma}\right) \rightarrow[0,1]$ :

$$
\hat{f}_{i}^{\beta}=f_{i}^{\beta} \cdot\left(h\left(\pi_{j \mid v_{i}^{\beta}}^{\gamma}\right) \cdot\left(\pi_{i}^{\beta}-1\right)+1\right) .
$$

where $\hat{f}_{i}^{\beta}$ is the estimated path flow, $f_{i}^{\beta}$ is the initial path flow, and $\pi_{i}^{\beta}$ is the flow distribution of path $v_{i}^{\beta}$. The influence of cascading effects for $v_{i}^{\beta}$ is determined by the stationary distribution $\pi_{j \mid v_{i}^{\beta}}^{\gamma}$. For example, in Figure 2 at hypervertex $v_{2}^{\gamma}$ a random walker is initialised, even if no of the underlying paths are interruped.

\section{Application}

The application presented in this section is used to demonstrate the usefulness of the methodology in addressing a specific problem. The application shows the design and implementation of a NiN model for estimating changes in traffic flow due to edge interruptions on two road networks. The first road network is the classical benchmark network of Sioux Falls, which is used within transport research to test, demonstrate and compare methods and algorithms. The second investigated road network is a real-world example from the region around the city of Chur, the capital of Grisons, the largest and easternmost canton of Switzerland.

In both cases, the initial traffic flow assignments were performed using a macroscopic traffic flow model. The networks were modelled as NiN and calibrated with the initial assignment results. Edges were randomly removed, and the traffic flows were estimated using diffusion processes as described above. To show the influence of the multi-layer approach, the same procedure was conducted with a single-layer network (SLN) model. The results of both modelling approaches were compared with the ground truth observed from the macroscopic traffic flow model with the same network topologies.

The remainder of this section is structured as follows. First, an overview of the networks and data used is given, followed by a description of the assumptions made during the modelling process. Finally, the implementation and simulation process is described in detail.

\section{Data}

Sioux falls

Although the Sioux Falls scenario is not considered realistic, it is used in many publications. Morlok et al. (1973) first introduced the network as a traffic equilibrium network. Later, the network was adapted as a benchmark and test scenario in many publications, including (LeBlanc et al. 1975; Suwansirikul et al. 1987; Meng et al. 2001; Chakirov and Fourie 2014). A more detailed list of use cases and the original data set is given by (Stabler et al. 2018).

The network was directed and consisted of 24 vertices and 76 edges, where each vertex also represented an origin-destination vertex, i.e. the area was divided into 24 zones. All network data including the vertex labels given in Fig. 3a were taken from (LeBlanc et al. 
a) Sioux Falls

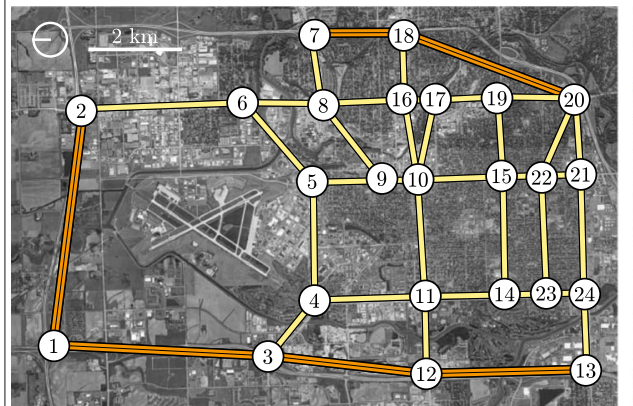

Vertex $\bar{\equiv}$ Motorway

Fig. 3 The infrastructure networks of Sioux Falls and Chur. a The Sioux Falls network is located in South Dakota, USA and consists of 24 vertices and 77 edges. Each vertex is associated with an origin and destination location. The edges are directed, i.e. there is an edge $(1,2)$ and $(2,1)$. b The Chur network is located in the canton of Grisons, Switzerland and consists of 1262 vertices and 3199 edges. The area is divided into 37 zones which represent the origin and destination locations

1975). Except for the vertex coordinates, which were taken from (Chakirov and Fourie 2014), since the original article does not contain this information. The origin-destination flows in the original article are given in thousands of vehicles per day with integer values up to 44, i.e. the origin-destination flows were the values from the table multiplied by 100 . They thus amount to 0.1 of the original daily flows and could, therefore, be considered as approximate hourly flows. This conversion was initially carried out to compare the objective values with the articles published in the 1980s and 1990s. The units of free flow travel time are $0.01 \mathrm{~h}$, but they are often interpreted as being minutes (Stabler et al. 2018). As an edge cost function the "traditional" BPR function proposed by the Bureau of (1964) was used:

$$
c_{e}:=t_{e}\left(x_{e}\right)=t_{e}^{0}\left(1+a_{e}\left(\frac{x_{e}}{y_{e}}\right)^{b_{e}}\right)
$$

where $t_{e}$ is the travel time at edge $e$ given the edge traffic flow $x_{e}, t_{e}^{0}$ is the free flow travel time, $y_{e}$ the edge capacity, and $a_{e}$ and $b_{e}$ are parameters for calibration, here chosen as $a_{e}=0.15$ and $b_{e}=4, \forall e \in \mathcal{E}$.

\section{Chur}

As a real-world example, the road network in the Rhine Valley around the city of Chur, Switzerland was investigated. An overview of the network is given in Fig. 3b. Only national, main and secondary roads were considered for the analysis. This corresponded to about $51 \mathrm{~km}$ of national roads, $165 \mathrm{~km}$ of main roads and $395 \mathrm{~km}$ of secondary roads. The network was represented as a directed graph with 1262 vertices and 3199 edges.

The information of the Chur road network was obtained from the VECTOR25 dataset of swisstopo (JD100042). This data set shows a complete national coverage and describes 8.5 million objects with their position, shape and their neighbourhood relations (topology) as well as the type of object and other special features. The accuracy of the geodata is in the range of 3 to $8 \mathrm{~m}$ and is available as ESRI shapefile for the Swiss coordinate system CH1903/LV03 LN02 (ESPG-Code: 21781). The road sections were described by their direction, length, free flow speed, capacity and the parameters of the edge cost function. 
For consistency reasons, the same cost function as in the Sioux Falls scenario [Eq. 8] was used.

The trips in the region were performed between 37 zones, based on judicial districts, as shown in Fig. 2b. All trips made from an origin to a destination zone in a given period were stored in an origin-destination matrix. Since there was not enough information available on the distribution of trips for the area of interest, a gravitational distribution model (de Dios Ortuozar and Willumsen 2011) was used to estimate the trips based on census data for each zone (e.g. demographics, households, work locations). The obtained gravitational model was calibrated by the Swiss national traffic model (FOSD 2015), which provided data for the motorway and main roads. In addition, data from traffic counting stations in the study area were used to calibrate the initial traffic assignment, by adapting the estimated origin-destination matrix.

\section{Assumptions}

In order to reduce complexity and apply the methodology to real problems, several assumptions were made during the modelling process. The assumptions and their justifications are listed below.

1 The objective was to model non-recurring situations such as road works or interruptions due to extreme weather events (e.g. floods, mud blockades). Although these events occur unexpectedly, it was assumed that travellers already had some information about the situation. In other words, it was not the traffic flow immediately after the event that was taken into account, but the one after a few days, so that travellers could adapt to the new situation. This also made it possible to compare the results with the results of the macroscopic traffic flow simulation, which assume an equilibrium between supply and demand in the interrupted scenario.

2 It was assumed that interrupted edges were removed entirely from the network, although it would be possible to consider capacity constraints (see "Discussion" section). As indicated under assumption number one, the interest was in extreme events where the effects could also be observed a few days later, so a complete road closure was chosen to express this severity.

3 It was assumed that there was no mode change, i.e. people who used a car in the initial configuration also used the car in the modified configuration, and did not change to another means of transport such as walking or cycling.

4. Due to the high complexity, not all possible paths were modelled. Instead, only the already selected paths and their $k$ most similar paths were considered. A similarity was determined using the BPR edge cost function [Eq. 8]. This means that the paths between origin and destination were evaluated by the travel costs observed in the baseline scenario. This approach ensured that very unlikely paths did not have to be considered.

5 Also to reduce complexity, it has been assumed that travellers can make a maximum of three decisions: (i) they can stay on the path initially chosen, (ii) they can switch to the next cheaper path or (iii) to the next more expensive one. This assumption is based on the assumption that travellers know about their paths and alternatives. In the baseline scenario, which is in equilibrium, there is no incentive 
to change paths, but in the interrupted scenario, travellers can speculate about using an initially better path with the risk of others using it, or an initially worse path that might be better under the new configuration. If a traveller is on an interrupted path, he has only the options (ii) or (iii). If the traveller is also on the best path in the baseline scenario, he just has the option (iii). The costs of the paths were determined using Eq. 8.

6 In order to model the cascading effects in the network, only paths where the original traffic load changed, were used, i.e. edges between pairs of origin-destination groups where there was no change were weighted by 0 and therefore were not considered in the diffusion process.

\section{Implementation}

\section{Initial traffic flow assignment}

The starting point was the simulation result of a classical macroscopic traffic flow model on an intact transportation network. Path statistics were generated from this data, i.e. the path flows were simulated and evaluated. Thereby, a path flow represents the quantitative amount of movements from an origin to a destination on a particular path. In the simplest case of a static user equilibrium, the traffic assignment problem is presented as follows:

$$
\min Z=\sum_{e} \int_{0}^{x_{e}} c_{e}(\omega) \mathrm{d} \omega
$$

subject to the demand and non-negativity constraints given by

$$
\begin{gathered}
\sum_{p} f_{p}^{o d}=q_{o d} \quad \forall p \in \mathcal{P}_{o d} \\
f_{p}^{o d} \geq 0
\end{gathered}
$$

where $x_{e}$ is the flow on edge $e$ comprised of the sum of flows on the paths sharing edge $e, c_{e}(\omega)$ is the cost on edge $e$ for a flow of $\omega, f_{p}^{o d}$ is the flow on path $p$ connecting origin $o$ and destination $d$ and $q_{o d}$ is the total traffic demand between $o$ and $d$.

Substantial research has been carried out on this problem and its extension to more practical approaches, including the representation of dynamic traffic phenomena such as queues, spillbacks, wave propagation, capacity drops and so on. In this work, this simplified approach was used due to the universality of the problem and its simple mathematical handling. In addition, the simple and commonly used Frank-Wolfe algorithm was used to solve the optimisation problem (Jayakrishnan et al. 1994; Chen et al. 2002). However, this does not affect the applicability of the proposed method, as it can be applied independently of the problem.

\section{NiN setup and failure propagation}

According to the proposed methodology, the basic elements in layer $\alpha$ were formed by the vertices of the infrastructure networks. The network paths in layer $\beta$ were obtained from the initial macroscopic traffic flow simulations. Paths with the same origin and destination are grouped in layer $\gamma$.

The contained pathways were ranked by travel costs [Eq. 8] and connected accordingly, i.e. the path with the lowest travel costs was located at the beginning of the Markov chain, followed by the path with the second lowest costs, and so on. As described in assumption 
number five, only the neighbouring paths were connected. To take into account alternative routes that were not used in the initial configuration, additional paths were added to each origin-destination group. These paths were generated using the Yen's algorithm (Yen 1970) to calculate the $k$-shortest loop-less paths for a graph with non-negative edge costs. These additional paths have been disabled, meaning that such a path could not be visited in the initial configuration, i.e. the edge weights and the initial traffic flow were set to 0 . In the top layer, $\delta$ all origin-destination groups were combined and connected based on their common road sections.

To initialise the failure propagation a set of edges were randomly removed from the infrastructure network. Care was taken to maintain the connectivity of the network such that there were no disconnected origin or destination vertices. Paths that contained these interrupted edges were disabled by setting the probability to zero that someone would visit or maintain on this path. With a certain probability, additional paths were enabled. Based on the new topology of the Markov chain and the (estimated) transition probabilities, the new stationary distributions were calculated. As a result of these changes in traffic flow, positive edge weights were assigned to edges in layer $\delta$. The second diffusion process was performed to initiate other redistribution processes at layer $\gamma$, as described in the methodology. This process continued until the traffic flow no longer changed.

Bayesian inference and MCMC methods were applied to estimate the transition probabilities for the diffusion process. This was done by minimising the normalised mean square errors of edge flows based on the results from the traffic flow simulations performed on the interrupted network topology where on edge was interrupted. For the parameter estimation (training process) 100 different network configurations were used.

\section{Single-layer network approach}

To evaluate the obtained results, a comparison between the proposed NiN model and a traditional complex network diffusion process on a single-layer network (SLN) was made. Since the traffic flows are edge properties, the initial topology of the road network has been converted into a higher-order network of order two (Rosvall et al. 2014; Scholtes 2017; Lambiotte et al. 2018), i.e. intersections were turned into edges and road sections into vertices (Porta et al. 2006). To consider the failures of the road sections, the incoming edges of the affected higher-order vertices were removed, i.e. in a diffusion process, the traffic flow can only flow away from these vertices. On this modified higher-order network, a diffusion process was carried out where the diffusion quantity corresponded to the traffic flows of the removed road sections. In other words, the travellers move away from the interrupted road sections. Finally, the new traffic flow configuration was mapped back to the initial road network to allow a comparison with the NiN model and the results of the traffic flow simulations.

\section{Technical implementation}

For reasons of rapid development and comparison, all the models were programmed in pure Python and executed on a single core. (Consequently, a considerable reduction of the computation time is to be expected by using compiled languages such as $\mathrm{C}++$ and utilising parallel computing.) The simulation runs were conducted on a single Intel Core i7-4770 CPU 3.40Ghz, 16GB DDR2 PC running on Linux 64 bit operating system (Ubuntu 16.04). A conjugate direct Frank-Wolfe (CFW) algorithm (Mitradjieva and Lindberg 2013), was 
implemented to solve the traffic assignment problem [Eq. 9]. The convergence criterion was set to a relative gap of $10^{-4}$. The network modelling for the NiN and the higher-order networks was done with the python package cnet.

\section{Results and discussion}

The results of the previously defined applications are discussed in this section in order to help illustrate the applications of the NiN model and map out the next steps for research. The presented approach allowed the estimation of the traffic flow by utilising modified multi-layer hypergraphs to model vertices themselves as networks. This allowed encoding multiple pieces of information such as the topology, paths used and origin-destination information, within one consistent graph structure.

Both the Sioux Falls scenario and the Chur scenario were both evaluated with a NiN model and an SLN model. As ground truth, the results of a macroscopic traffic flow simulation were used to determine the errors of the traffic flow estimation. In order to assess the behaviour in case of edge failure, 1 to 15 random edges were removed from the infrastructure network. With the help of diffusion processes, the traffic flow changes of both approaches were estimated and compared with the (exact) result of the traffic flow simulation. In the Sioux Falls scenario, the relative deviations of the traffic flows were compared. This was not possible in the Chur scenario, therefore, the relative differences were used for the analysis. In both scenarios, it could be shown that the NiN model provides better estimates than the SLN model.

In the remainder of this section, the two scenarios and their results will be discussed in more detail, followed by a general discussion about the observations and future improvements.

\section{Sioux falls scenario}

Due to the design and size of the Sioux Falls scenario, traffic flows were observed on each road section in the initial configuration. Figure 4a illustrates the initial flow by the varying line width, which can also be interpreted as an impotence measure,

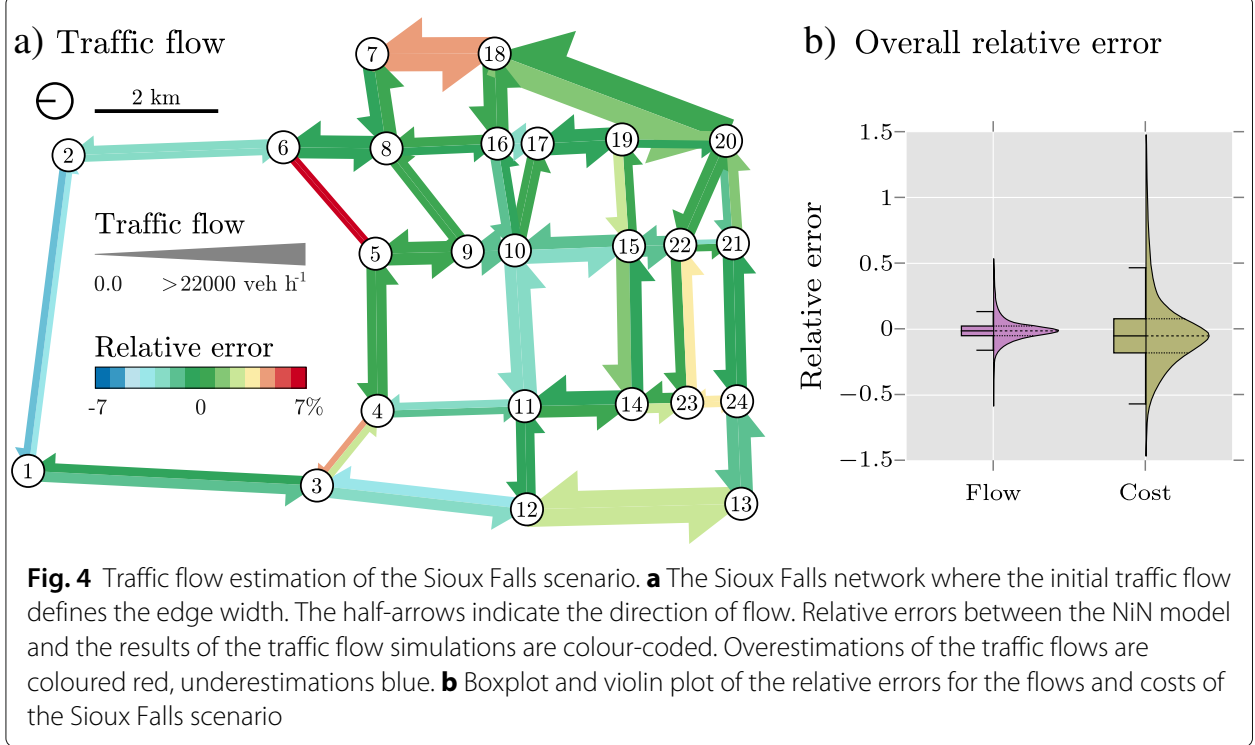


i.e. higher flows indicate more critical connections. The average relative error between the NiN model and the macroscopic traffic flow model [Eq. 9] is colour-coded, with positive (red) values representing overestimation and negative (blue) values representing underestimation. Figure 4a shows the average relative error for a scenario where a single edge was randomly removed. It can be observed that the flow near the centre (e.g. near the vertices $10,15,16,17,19$ ) is well estimated (less than 2.5\%). In addition, the estimates for edges with a high initial traffic flow are just as good (e.g. Edge $(20,18))$. Edge $(5.6)$ shows the highest overestimation at $6.5 \%$, while Edge $(2.1)$ underestimates the traffic flow by $4.1 \%$, resulting in both edges having a low initial traffic flow. The average total relative flow error for all scenarios in which an edge was randomly removed is $-2.7 \%(\sigma=0.064)$, and $95 \%$ of the estimated values are in the $+13.4 \%$ and $-15.9 \%$ ranges, as shown in Fig. 4 b. Since the implemented cost function [Eq. 8] considers the estimated traffic with the performance of $b_{e}=4$, the cost estimate shows higher uncertainties, i.e. $95 \%$ of the cost estimates are in the range between $+46.7 \%$ and $-56.6 \%$.

If the NiN model is compared with the SLN model, there are hardly any deviations if only a few edges are randomly removed. However, if several edges are removed, the SLN model begins to underestimate the traffic flow (see Table 1 and Fig. 5). This is caused by the redistribution of the traffic flow due to the diffusion process. Since the traffic flow is not constrained by an origin and destination pair, travellers can diffuse to all edges in the network, which assigns traffic flows more evenly over the whole network. In contrast, the traffic flow in the NiN model can only be redistributed to other paths with the same origin and destination, so that traffic in the network cannot spread too far. However, an overor underestimation can occur if too many or too few travellers are assigned to the new paths or if paths are taken which were not considered by the $k$-shortest paths. Figure 5 also shows that the distribution of the relative error is more symmetrically allocated for the NiN model than for the SLN model, which tend to underestimate the traffic flows.

Table 1 Average relative errors and standard deviations of the Sioux Falls scenario when multiple edges are removed

\begin{tabular}{llllll}
\hline No. of rem. edges & \multicolumn{2}{l}{ SLN model } & & \multicolumn{2}{l}{ NiN model } \\
\cline { 2 - 3 } & mean & std. & & mean & std. \\
\hline 1 & -0.036 & 0.077 & & -0.027 & 0.064 \\
2 & -0.054 & 0.107 & & -0.040 & 0.097 \\
3 & -0.063 & 0.126 & -0.046 & 0.115 \\
4 & -0.073 & 0.148 & -0.050 & 0.130 \\
5 & -0.082 & 0.170 & -0.057 & 0.149 \\
6 & -0.092 & 0.181 & -0.060 & 0.161 \\
7 & -0.100 & 0.194 & -0.066 & 0.172 \\
8 & -0.108 & 0.207 & -0.070 & 0.178 \\
9 & -0.116 & 0.223 & -0.072 & 0.187 \\
10 & -0.118 & 0.247 & -0.072 & 0.193 \\
11 & -0.121 & 0.259 & -0.073 & 0.203 \\
12 & -0.127 & 0.276 & -0.076 & 0.201 \\
13 & -0.141 & 0.280 & -0.083 & 0.212 \\
14 & -0.141 & 0.288 & -0.083 & 0.212 \\
15 & -0.142 & 0.295 & -0.084 & 0.213 \\
\hline
\end{tabular}




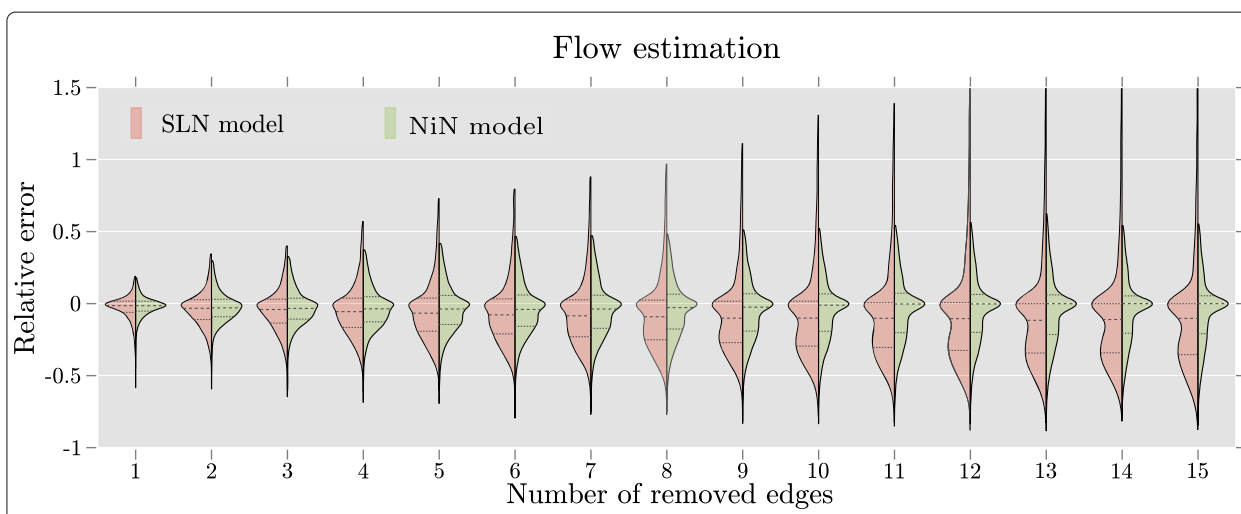

Fig. 5 Violin plot of the relative errors for the Sioux Falls scenario when multiple edges are removed. In red (left) the kernel density of the SLN model is shown, while in green (right) the kernel density of the NiN model is shown. The number of removed edges ranges from 1 to 15 , corresponding to a removal of $1.3 \%$ to $19.7 \%$ of the edges in the network. Dotted lines within the densities represent the $25 \%$ and $75 \%$ quantiles, respectively, while the dashed lines represent the median values

\section{Chur scenario}

In contrast to the Sioux Falls scenario where traffic flows occurred on every road section, the Chur scenario had road sections that were not used in the initial traffic configuration. To compare deviations from the true value of zero, instead of the relative error measure, the relative difference measure was used, which compared the difference between two values to their average magnitude:

$$
d_{r}\left(x_{e}, \hat{x}_{e}\right)=2 \frac{\hat{x}_{e}-x_{e}}{\left|\hat{x}_{e}\right|+\left|x_{e}\right|}
$$

This is a signed expression, positive when the estimated edge traffic flow $\hat{x}_{e}$ exceeds the observed edge traffic flow $x_{e}$ and negative when $x_{e}$ exceeds $\hat{x}_{e}$. Its value always lies between -2 and 2, i.e. if a flow is assigned to an edge where the initial flow is 0 the relative difference is 2 . By using absolute values in the denominator, negative numbers are handled in a reasonable way.

Both Figs. 6 and 7 clearly show that an SLN model approach to estimate traffic flow changes in a real-world network leads to misrepresentations of the actual traffic flow by assigning flows to edges which are not in use. In other words, travellers were assigned to edges, which were not part of feasible paths between the desired origin and destination locations. The NiN model showed no such behaviour, as travellers were only allowed to change their paths while retaining their initial origin and destinations.

In particular, this overestimation can be observed in the city centres of Chur and Domat-Ems, using the SLN model. Access roads and side roads are wrongly estimated. Since the original macroscopic traffic flow model is a regional model, i.e. no traffic flow is assigned to these secondary roads. This could change if a more detailed model is used (e.g. microscopic model).

Increasing the number of removed edges also led to an overestimation of traffic flow in the NiN model, as shown in Fig. 6b. Instead of exploring and accounting new paths between the origins and destinations, the NiN model assigned more flows to the existing paths. This could be due to the model calibration, which was done on a network where only one edge was randomly removed and not several edges. 


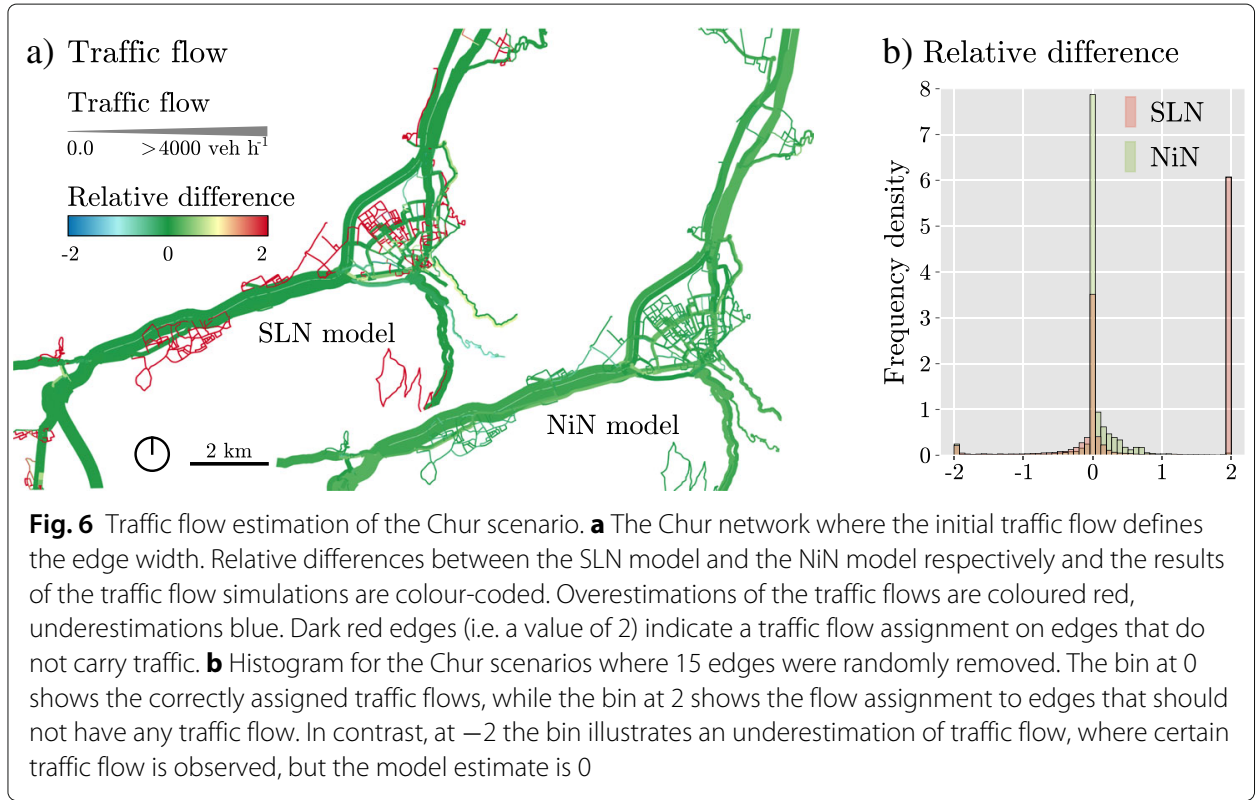

\section{Discussion}

In both scenarios, the NiN model performed better than the SLN model. This can be attributed to the fact that the NiN model takes into account the path statistics and the origin and destination locations in addition to the network topology. This was clearly shown in the Chur scenario where the SLN model had assigned traffic flows to roads where none should be. Consequently, if one wants to investigate dynamic processes on transport networks using a complex network approach, this should be taken into account, otherwise, it could lead to wrong conclusions.

Since the NiN model is able to encode multiple information, more data has to be provided to create such a model. While an SLN model only requires the network topology,

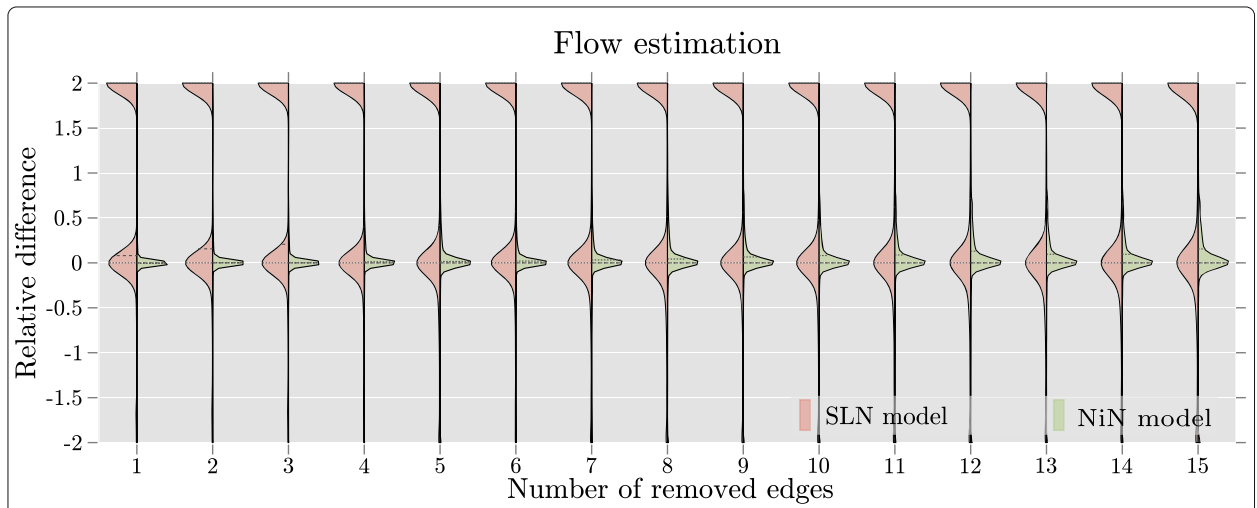

Fig. 7 Violin plot of the relative differences for the Chur scenario when multiple edges are removed. In red (left) the kernel density of the SLN model is shown, while in green (right) the kernel density of the NiN model is shown. The number of removed edges ranges from 1 to 15 . Dotted lines within the densities represent the $25 \%$ and $75 \%$ quantiles, respectively, while the dashed lines represent the median values. Due to the kernel density estimation, overestimates are presented continuously around the value of 2 , while in reality, this corresponds to a point distribution, as shown in Fig. $6 \mathrm{~b}$ 
a NiN model also requires path statistics. This is a particular problem for historical networks where such data is no longer available. However, due to increasing digitalisation (GPS tracking, telephone data, Twitter data) and advancing development in traffic models, more and more such data is nowadays available for research (e.g. Song et al. (2010); Tang et al. (2015); Jurdak et al. (2015)).

The introduced NiN model represents the first step to represent complex dynamic processes on transport networks using complex network approaches. For example, the decision of which path to take is reduced to a simple diffusion process instead of solving a complex optimisation problem. Naturally, this approach does not provide the same accuracy as classical traffic flow models, but it allows general statements to be made with relatively simple means, e.g. showing the cascading effects of several edge failures in the network.

In this example, some assumptions were made to reduce the complexity of the problem. However, this also led to a reduction in accuracy and applicability. In future work, some of these assumptions could be relaxed. For example, it was assumed that interrupted edges were removed entirely from the network. However, it would be possible to consider capacity reductions similarly as the cascading effects were studied, i.e. instead of removing the edges, a path diffusion process is performed, and the traffic flow corresponding to the capacity reduction is redistributed. This implementation would represent a more realistic representation of the real weld and thus significantly extend the application area of the model. In addition, it was assumed that travellers could only make three choices: (i) they could stay on the path initially chosen, (ii) they could switch to the next cheaper path or (iii) to the next more expensive one. This simplifies the estimation of the parameters but limits reality. Especially in the context of real observations, it would be interesting to see how travellers behave in a disturbance and what alternative paths they take.

If real-world observations are not available, another improvement would be the extension to an underlying microscopic traffic flow modelling approach (e.g. agent-based approach), to reproduce the real-world behaviour of the daily path of individuals in an urban environment. Thereby individual agents choose activities at different locations. Sequences of activities are generated and equilibrated based on a co-evolutionary algorithm that alters the agent's behaviour from iteration to iteration. The objective is to find optimal routes, modes and departure times in order to maximise the total utilities of the agents' daily activity schedules (Chakirov and Fourie 2014; Horni et al. 2016). In contrast to the aggregated path flows from a macroscopic point of view, where each path is assigned to an individual agent. The setup of the NiN would be similar, but the underlying model would provide additional details.

\section{Conclusions}

Understanding traffic flow in urban areas has great importance and implications from an economic, social and environmental point of view. For this reason, numerous disciplines are working on this topic. Although complex network theory made their appearance in transportation research through empirical measures, little research has so far been done to investigate the relationship between dynamic traffic patterns and the underlying structures of the transportation networks.

In this work, the application of a novel Networks in Networks (NiN) approach is presented to study changes in traffic flow caused by topological changes in the transportation 
network, from a complex network perspective. The NiN structure is a special type of multi-layer network in which vertices are networks themselves. This embedded network structure makes it possible to encode multiple pieces of information such as topology, paths, and origin-destination information, within one consistent graph structure. Since each vertex is an independent network in itself, it is possible to implement multiple diffusion processes with different physical meanings. In this way, it is possible to estimate how the travellers' paths will change and to determine the cascading effect in the network.

To test the capabilities and gain insights into real-world systems, the model was applied to two complex infrastructure systems, the Sioux Falls benchmark network and a realworld road network in Switzerland. The networks were modelled as NiN and calibrated with initial traffic flows from a macroscopic model. Edges were randomly removed, and the traffic flows were estimated using diffusion processes. To show the influence of the multi-layer approach, the same procedure was conducted with a single-layer network (SLN) model. The results of both modelling approaches were compared with the ground truth observed from the macroscopic traffic flow model. In both scenarios, the NiN model performed better than the SLN model. Especially for the real-world network, the NiN model gives better results, since in addition to the network topology also path statistics and the origin and destination information were taken into account.

The work presented here is the first step towards a better understanding of complex dynamic processes in transport networks using approaches from complex network theory. Naturally, this approach does not provide the same accuracy as classical traffic flow models, but it allows general statements to be made with relatively simple means. In addition, NiN's uniform graph structure offers a new perspective on the problem of traffic assignment that goes beyond classical approaches. For example, while classical traffic flow models evaluate the (user) equilibrium by solving an optimisation problem, the proposed method uses a (path) diffusion process instead. This is computationally more efficient and should be explored in future research. Furthermore, the presented approach is limited to the estimation of traffic flow changes caused by multiple edge failures, given an initial traffic flow configuration. Future research should focus on further developing this approach to address relevant issues such as mode changes, temporal changes, spillbacks and gridlock effects.

\section{Nomenclature}

$\begin{aligned} G & \text { network graph } \\ \mathcal{E} & \text { set of edges of the network } \\ \mathcal{V} & \text { set of vertices of the network } \\ e & \text { edges in the network } \\ v, o, d & \text { vertices in the network } \\ o & \text { origin vertex } \\ d & \text { destination vertex } \\ p & \text { specific path in the network } \\ \mathcal{P} & \text { set of all nonempty paths } \\ \mathcal{P}_{\text {od }} & \text { set of all od-paths } \\ \mathcal{L} & \text { set of layers } \\ \alpha, \beta, \gamma, \delta & \text { layers of the network } \\ \mathcal{G} & \text { set of graphs }\end{aligned}$




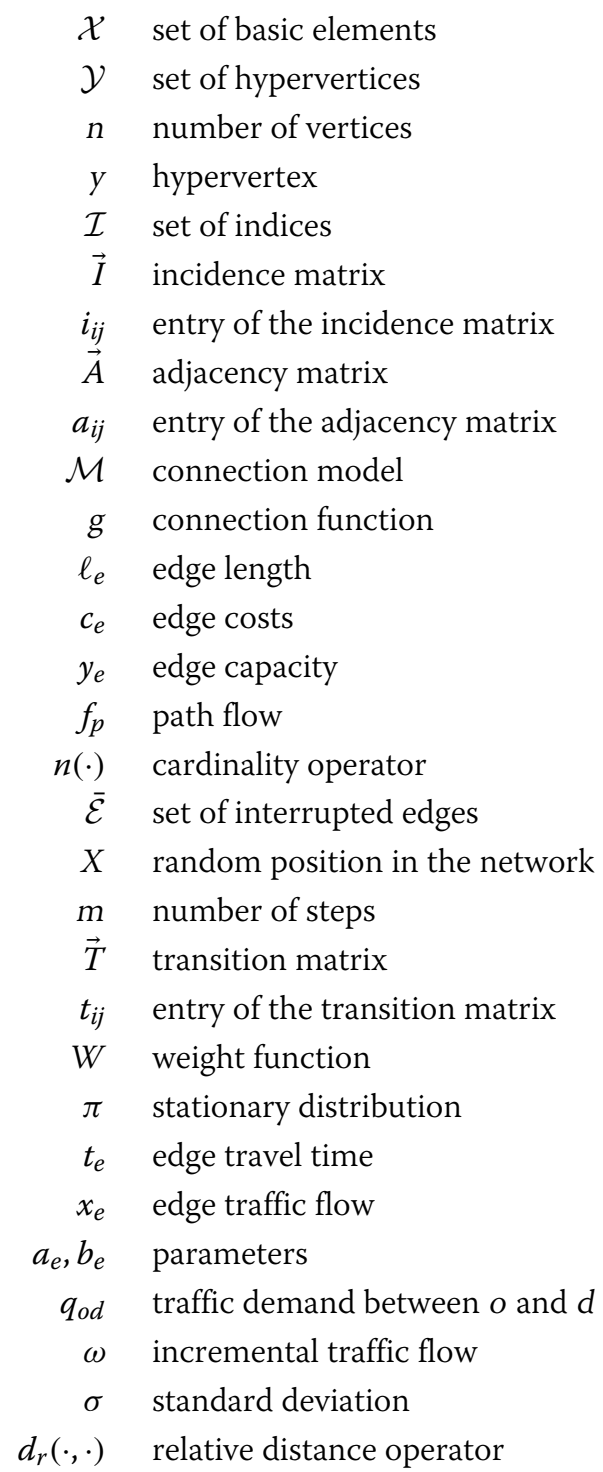

\section{Endnotes}

${ }^{1}$ In a classical single-layer hypergraph $\mathcal{X}_{M}$ usually refers to hyperedges, which are finite sets of basic elements (vertices) representing their relationships (connections). In the scope of a multi-layer hypergraph, the vertices of each layer are defined as the hyperedges of the previous ones. To avoid confusion in terminology, such higher-order elements are referred to as hypervertices or vertices in this work.

${ }^{2} \mathrm{~A}$ division by $\sum_{k} W\left(v_{i}, v_{k}\right)$ is needed to normalise the values such that $\sum_{j} W\left(v_{i}, v_{j}\right)=1$.

\section{Acknowledgements}

The authors would like to thank the editor and the anonymous reviewers for their constructive comments and valuable suggestions to improve the quality of the article.

Funding

The work presented here has received funding from the European Union's Seventh Programme for Research, Technological Development and Demonstration under grant agreement no. 603960, and from Horizon 2020, the European Union's Framework Programme for Research and Innovation, under grant agreement no. 636285.

\section{Availability of data and materials}

Particular emphasis was put on using open data wherever possible. Data sets used are as follows: the Sioux Falls transportation network, including the topology, trips and estimated traffic flows is available under https://github.com/ 
bstabler/TransportationNetworks (last access: 14 November 2018). The geodata for Grisons can be found at the geographic information system of the canton of Grisons, accessible via https://geogr.ch/ (last access: 14 November 2018). Topographic data such as the administrative borders of the municipalities of Switzerland, aerial imagery and the road network from Switzerland are provided by https://www.swisstopo.admin.ch/ (last access: 14 November 2018) and obtained from https://opendata.swiss/ (last access: 14 November 2018). The python package for the network modelling is available under https://github.com/hackl/cnet. Detailed information about the data used in this work is available upon request.

\section{Authors' contributions}

$\mathrm{JH}$ developed the theoretical formalism, designed the model and the computational framework, and performed the numerical simulations. Both $\mathrm{JH}$ and BTA authors contributed to the final version of the manuscript. BTA supervised the project. Both authors read and approved the final manuscript.

Ethics approval and consent to participate

Not applicable.

Consent for publication

Not applicable.

\section{Competing interests}

The authors declare that they have no competing interests.

\section{Publisher's Note}

Springer Nature remains neutral with regard to jurisdictional claims in published maps and institutional affiliations.

Received: 14 November 2018 Accepted: 1 May 2019

Published online: 31 May 2019

\section{References}

Albert R, Jeong H, Barabási AL (1999) Internet: Diameter of the World-Wide Web Despite. Nature 401 (6749):130-131. https://doi.org/10.1038/43601.43601

Bao J, Chen W, Shui Y-s, Xiang Z-t (2017) Complexity analysis of traffic time series based on multifractality and complex network. In: 2017 4th International Conference on Transportation Information and Safety (ICTIS). IEEE, Piscataway. pp 257-263. https://doi.org/10.1109/ICTIS.2017.8047774. http://ieeexplore.ieee.org/document/8047774/

Barabási AL, Albert R (1999) Emergence of Scaling in Random Networks. Science 286(5439):509-512. https://doi.org/10 $1126 /$ science. 286.5439 .509

Barrat A, Barthelemy M, Vespignani A (2008) Dynamical Processes on Complex Networks. Cambridge University Press, Cambridge. https://doi.org/10.1017/CBO9780511791383

Barthélemy M (2011) Spatial networks. Phys Rep 499(1-3):1-101. https://doi.org/10.1016/j.physrep.2010.11.002

Bavelas A (1950) Communication Patterns in Task-Oriented Groups. J Acoust Soc Am 22(6):725-730. https://doi.org/10. $1121 / 1.1906679$

Bianconi G (2018) Multilayer Networks: Structure and Function. Oxford University Press, Oxford

Boccaletti S, Bianconi G, Criado R, del Genio Cl, Gómez-Gardeñes J, Romance M, Sendiña-Nadal I, Wang Z, Zanin M (2014) The structure and dynamics of multilayer networks. Phys Rep 544(1):1-122. https://doi.org/10.1016/j.physrep.2014.07. 001

Brin S, Page L (1998) The Anatomy of a Large-Scale Hypertextual Web Search Engine. In: Seventh International World-Wide Web Conference (WWW 1998). pp 1-20

Buchhold V, Sanders P, Wagner D (2018) Real-Time Traffic Assignment Using Fast Queries in Customizable Contraction Hierarchies. In: D'Angelo G (ed). 17th International Symposium on Experimental Algorithms (SEA 2018), Schloss Dagstuhl-Leibniz-Zentrum fuer Informatik, Dagstuhl, Germany, Leibniz International Proceedings in Informatics (LIPICS), vol. 103. pp 27:1--27:15

Bureau of PublicRoads (1964) Traffic Assignment Manual. Manual, Urban Planning Division. US Department of Commerce, Washington

Cardillo A, Gomez-Gardeñes J, Zanin M, Romance M, Papo D, Del Pozo F, Boccaletti S (2013) Emergence of network features from multiplexity. Sci Rep 3:1-6. https://doi.org/10.1038/srep01344

Chakirov A, Fourie PJ (2014) Enriched Sioux Falls Scenario with Dynamic and Disaggregate Demand, Working paper, Future Cities Laboratory, Singapore - ETH Centre (SEC), Singapore. IVT, ETH Zurich. https://doi.org/10.3929/ethz-b000080996

Charypar D, Nagel K (2005) Generating complete all-day activity plans with genetic algorithms. Transportation 32(4):369-397

Chen A, Jayakrishnan R, Tsai WK (2002) Faster Frank-Wolfe Traffic Assignment with New Flow Update Scheme. J Trans Eng 128(1):31

Clauset A, Shalizi CR, Newman MEJ (2009) Power-Law Distributions in Empirical Data. SIAM Rev 51(4):661-703

Cliff AD, Haggett P, Ord K (1979) Graph theory and geography. In: Wilson RJ, Beineke LW (eds). Applications of Graph Theory. Academic Press, London. pp 293-326

Coelho R, Néda Z, Ramasco JJ, Augusta Santos M (2005) A family-network model for wealth distribution in societies. Physica A: Stat Mech Appl 353(1-4):515-528. https://doi.org/10.1016/j.physa.2005.01.037

Cozzo E, de Arruda GF, Rodrigues FA, Moreno Y (2016) Multilayer Networks: Metrics and Spectral Properties. In: Garas A (ed). Interconnected Networks. Springer International Publishing, Cham. pp 17-35. chap 2. https://doi.org/10.1007/ 978-3-319-23947-7_2 
Daganzo CF, Geroliminis N (2008) An analytical approximation for the macroscopic fundamental diagram of urban traffic. Trans Res Part B: Methodol 42(9):771-781

de Dios Ortuozar J, Willumsen LG (2011) Modelling transport. 4th edn. Wiley, West Sussex

De Montis A, Barthélemy M, Chessa A, Vespignani A (2007) The Structure of Interurban Traffic: A Weighted Network Analysis. Environ Plan B: Plan Des 34(5):905-924. https://doi.org/10.1068/b32128

Delling D, Sanders P, Schultes D, Wagner D (2009) Engineering Route Planning Algorithms. In: Lerner J, Wagner D, Zweig KA (eds). Algorithmics of Large and Complex Networks: Design, Analysis, and Simulation. Springer Berlin Heidelberg, Berlin. pp 117-139

Dettmann CP, Georgiou O (2016) Random geometric graphs with general connection functions. Phys Rev E 93(3):032,313

Erath A, Löchl M, Axhausen KW (2009) Graph-Theoretical Analysis of the Swiss Road and Railway Networks Over Time. Netw Spat Econ 9(3):379-400

Erath AL (2011) Vulnerability assessment of road transport infrastructure. ETH Zurich, Zurich. https://doi.org/10.3929/ ethz-a-006806584

Ferrario E, Pedroni N, Zio E (2016) Evaluation of the robustness of critical infrastructures by Hierarchical Graph representation, clustering and Monte Carlo simulation. Reliab Eng Syst Saf 155:78-96

FOSD (2015) Nationales Personenverkehrsmodell des UVEK, Aktualisierung auf den Basiszustand 2010. Report, Federal Office for Spatial Development (FOSD), Bern

Freeman LC (1977) A Set of Measures of Centrality Based on Betweenness. Sociometry 40(1):35

Geisberger R, Sanders P, Schultes D, Delling D (2008) Contraction Hierarchies: Faster and Simpler Hierarchical Routing in Road Networks. In: McGeoch CC (ed). Experimental Algorithms. Springer Berlin Heidelberg, Berlin. pp 319-333

Gentile G (2014) Local User Cost Equilibrium: a bush-based algorithm for traffic assignment. Transportmetrica A: Transp Sci 10(1):15-54

Gómez C, Sanchez-Silva M, Dueñas-Osorio L, Rosowsky D (2013) Hierarchical infrastructure network representation methods for risk-based decision-making. Struct Infrastruct Eng 9(3):260-274

Guo XI, Lu ZM (2016) Urban Road Network and Taxi Network Modeling Based on Complex Network Theory. J Inf Hiding Multimedia Signal Proc 7(3):558-568

Hackl J, Adey BT (2017) Generation of Spatially Embedded Random Networks to Model Complex Transportation Networks. In: Caspeele R, Taerwe L, Proske D (eds). 14th International Probabilistic Workshop. Springer International Publishing, Cham. pp 217-230

Hackl J, Adey BT (2018) Modelling multi-layer spatially embedded random networks. J Complex Netw 00(00):1-27

Hackl J, Adey BT, Lethanh N (2018a) Determination of Near-Optimal Restoration Programs for Transportation Networks Following Natural Hazard Events Using Simulated Annealing. Comput-Aided Civil Infrastruct Eng 33(8):618-637

Hackl J, Lam JC, Heitzler M, Adey BT, Hurni L (2018b) Estimating network related risks: A methodology and an application in the transport sector. Nat Hazards Earth Syst Sci 18(8):2273-2293

Heywood P, Maddock S, Casas J, Garcia D, Brackstone M, Richmond P (2018) Data-parallel agent-based microscopic road network simulation using graphics processing units. Simul Model Pract Theory 83:188-200

Hoogendoorn SP, Knoop V (2012) Traffic flow theory and modelling. In: van Wee B, Annema JA, Banister D (eds). The Transport System and Transport Policy: An Introduction. Edward Elgar Publishing Limited, Cheltenham. pp 125-159. chap 7

Horni A, Nagel K, Axhausen K (eds) (2016) The Multi-Agent Transport Simulation MATSim. Ubiquity Press, London

lacovacci J, Bianconi G (2016) Extracting information from multiplex networks. Chaos 26(6). https://doi.org/10.1063/1. 4953161

Jayakrishnan R, Tsai WT, Prashker JN, Rajadhyaksha S (1994) A faster path-based algorithm for traffic assignment. Transportation Studie

Jeub LGS, Balachandran P, Porter MA, Mucha PJ, Mahoney MW (2015) Think locally, act locally: Detection of small, medium-sized, and large communities in large networks. Phys Rev E 91(1):012,821. https://doi.org/10.1103/PhysRevE. 91.012821

Jurdak R, Zhao K, Liu J, AbouJaoude M, Cameron M, Newth D (2015) Understanding Human Mobility from Twitter. PLoS ONE 10(7):e0131,469

Kivelä M, Arenas A, Barthelemy M, Gleeson JP, Moreno Y, Porter MA (2014) Multilayer networks. J Complex Netw 2(3):203-271. https://doi.org/10.1093/comnet/cnu016

Lambiotte R, Rosvall M, Scholtes I (2018) Understanding Complex Systems: From Networks to Optimal Higher-Order Models:1-11. tbd. https://doi.org/1806.05977

Latora V, Marchiori M (2002) Is the Boston subway a small-world network?. Physica A: Stat Mech Appl 314(1-4):109-113. https://doi.org/10.1016/S0378-4371(02)01089-0

LeBlanc L, Morlok EK, Pierskalla WP (1975) An efficient approach to solving the road network equilibrium traffic assignment problem. Transp Res 9(5):309-318

Lighthill MJ, Whitham GB (1955) On Kinematic Waves. II. A Theory of Traffic Flow on Long Crowded Roads. Proc R Soc A: Math Phys Eng Sci 229(1178):317-345

Lim HW, Song J, Kurtz N (2015) Seismic reliability assessment of lifeline networks using clustering-based multi-scale approach. Earthq Eng Struct Dyn 44(3):355-369

Lin J, Ban Y (2013) Complex Network Topology of Transportation Systems. Transp Rev 33(6):658-685

Marr C, Hütt MT (2005) Topology regulates pattern formation capacity of binary cellular automata on graphs. Physica A: Stat Mech Appl 354(1-4):641-662. https://doi.org/10.1016/j.physa.2005.02.019

Masuda N, Porter MA, Lambiotte R (2017) Random walks and diffusion on networks. Phys Rep 716-717:1-58. https://doi. org/10.1016/j.physrep.2017.07.007

Meester R, Roy R (1996) Continuum Percolation. Cambridge Tracts in Mathematics, Cambridge University Press, Cambridge

Meng Q, Yang H, Bell MGH (2001) An equivalent continuously differentiable model and a locally convergent algorithm for the continuous network design problem. Transp Res Part B: Methodol 35(1):83-105

Mitradjieva M, Lindberg PO (2013) The Stiff Is Moving —Conjugate Direction Frank-Wolfe Methods with Applications to Traffic Assignment *. Transp Sci 47(2):280-293 
Moreno Y, Nekovee M, Pacheco AF (2004) Dynamics of rumor spreading in complex networks. Phys Rev E 69(6):066,130. https://doi.org/10.1103/PhysRevE.69.066130

Morlok EK, Schofer JL, Pierskalla WP, Marsten RE, Agarwal SK, Stoner JW, Edwards JL, LeBlanc LJ, Spacek DT (1973) Development and Application of a Highway Network Design Model. Report DOT-PH-11. Northwestern University, Evanston

Newman MEJ (2002) Spread of epidemic disease on networks. Phys Rev E 66(1):016,128. https://doi.org/10.1103/ PhysRevE.66.016128

Newman MEJ (2006) Finding community structure in networks using the eigenvectors of matrices. Phys Rev E 74(3):036,104. https://doi.org/10.1103/PhysRevE.74.036104

Parsonage E, Roughan M (2017) Fast Generation of Spatially Embedded Random Networks. IEEE Trans Netw Sci Eng 4(2):112-119. https://doi.org/10.1109/TNSE.2017.2681700

Porta S, Crucitti P, Latora V (2006) The network analysis of urban streets: A dual approach. Physica A: Stat Mech Appl 369(2):853-866. https://doi.org/10.1016/j.physa.2005.12.063

Rodrigue JP, Comtois C, Slack B (2009) The geography of transport systems. 2nd edn, Routledge

Rosvall M, Esquivel AV, Lancichinetti A, West JD, Lambiotte R (2014) Memory in network flows and its effects on spreading dynamics and community detection. Nat Commun 5:1-13. https://doi.org/10.1038/ncomms5630

Salnikov V, Schaub MT, Lambiotte R (2016) Using higher-order Markov models to reveal flow-based communities in networks. Sci Rep 6:1-13. https://doi.org/10.1038/srep23194

Schlögl M, Richter G, Avian M, Thaler T, Heiss G, Lenz G, Fuchs S (2019) On the nexus between landslide susceptibility and transport infrastructure - an agent-based approach. Nat Hazards Earth Syst Sci 19(1):201-219

Scholtes I (2017) When is a Network a Network?. In: Proceedings of the 23rd ACM SIGKDD International Conference on Knowledge Discovery and Data Mining - KDD '17. ACM Press, New York, New York. pp 1037-1046. https://doi.org/10. $1145 / 3097983.3098145$

Scholtes I, Wider N, Pfitzner R, Garas A, Tessone CJ, Schweitzer F (2014) Causality-driven slow-down and speed-up of diffusion in non-Markovian temporal networks. Nat Commun 5:5024. https://doi.org/10.1038/ncomms6024

Sen P, Dasgupta S, Chatterjee A, Sreeram PA, Mukherjee G, Manna SS (2003) Small-world properties of the Indian railway network. Phys Rev E 67(3):36,106

Smith DMD, Onnela JP, Lee CF, Fricker MD, Johnson NF (2011) Network Automata: Coupling Structure and Function in Dynamic Networks. Adv Complex Syst 14(03):317-339. https://doi.org/10.1142/S0219525911003050

Solé-Ribalta A, Gómez S, Arenas A (2016) A model to identify urban traffic congestion hotspots in complex networks. Royal Soc Open Sci 3(10):160,098. https://doi.org/10.1098/rsos.160098

Song C, Qu Z, Blumm N, Barabási AL (2010) Limits of Predictability in Human Mobility. Science 327(5968):1018-1021

Song X, Xie Z, Xu Y, Tan G, Tang W, Bi J, Li X (2017) Supporting real-world network-oriented mesoscopic traffic simulation on GPU. Simul Model Pract Theory 74:46-63

Stabler B, Bar-Gera H, Sall E (2018) Transportation Networks for Research

Suwansirikul C, Friesz TL, Tobin RL (1987) Equilibrium Decomposed Optimization: A Heuristic for the Continuous Equilibrium Network Design Problem. Transp Sci 21(4):254-263

Taaffe EJ, Gauthier HL, Morton O (1973) Geography of Transportation. Prentice-Hall Foundations of Economic Geography Series. Prentice-Hall, Upper Saddle River

Tang J, Wang Y, Liu F (2013) Characterizing traffic time series based on complex network theory. Physica A: Stat Mech App 392(18):4192-4201

Tang J, Liu F, Wang Y, Wang H (2015) Uncovering urban human mobility from large scale taxi GPS data. Physica A: Stat Mech Appl 438:140-153

Toroczkai Z (2005) Complex Networks. Los Alamos Sci 29:94-109

Vespignani A (2010) Complex networks: The fragility of interdependency. Nature 464(7291):984-985

Vugrin ED, Turnquist MA, Brown NJK (2014) Optimal recovery sequencing for enhanced resilience and service restoration in transportation networks. Int J Crit Infrastruct 10(3-4):218-246

Wang WX, Wang BH, Yin CY, Xie YB, Zhou T (2006) Traffic dynamics based on local routing protocol on a scale-free network. Phys Rev E 73(2):026,111

Wang Y, Szeto WY, Han K, Friesz TL (2018) Dynamic traffic assignment: A review of the methodological advances for environmentally sustainable road transportation applications. Transp Res Part B: Methodol 111:370-394

Wardrop JG (1952) Some Theoretical Aspects of Road Traffic Research. Proc Inst Civ Eng 1(3):325-362

Wasserman S, Faust K (1994) Social Network Analysis: Methods and Applications. Structural Analysis in the Social Sciences. Cambridge University Press, New York

Watts DJ, Strogatz SH (1998) Collective dynamics of 'small-world' networks. Nature 393(6684):440-442

Yan Y, Zhang S, Tang J, Wang X (2017) Understanding characteristics in multivariate traffic flow time series from complex network structure. Physica A: Stat Mech Appl 477:149-160

Yen JY (1970) An algorithm for finding shortest routes from all source nodes to a given destination in general networks. Q Appl Math 27(4):526-530 